\title{
Three-dimensional analysis of pulp chambers in mandibular first deciduous molars
}

\author{
Hiroko Agematsu, Masaharu Ohnishi, Satoru Matsunaga, Hideki Saka, \\ Ken Nakahara and Yoshinobu Ide \\ Department of Anatomy, Tokyo Dental College \\ 1-2-2 Masago, Mihama-ku, Chiba 261-8502, JAPAN
}

\begin{abstract}
In crown restoration, it is important to understand the internal anatomical feature of each tooth, as the morphology of human deciduous teeth is particularly complex. The purpose of this study was to clarify threedimensionally the topographic relationship between the crown contour and the pulp chamber of the mandibular first deciduous molars using a micro-CT system. Fifteen mandibular first deciduous molars obtained from the dried skulls of Indian children in the deciduous dentition period were used as specimens. Three-dimensional reconstructed images were reproduced by combining the two-dimensional slice imaging data using a micro-CT system. Crown contours and shapes of the pulp chamber were observed from various directions. In addition, the thickness of tooth substance at each pulp horn was also measured. In observations of the topographic relationship between the crown contour and the pulp chamber, the pulp horn of the mesiobuccal pulp chamber was found to project the most to the cusp side. The distance between the pulp horn and enamel surface was also shortest $(2.45 \pm 0.30 \mathrm{~mm})$ in this region. Furthermore, in teeth with a deep, wide trigonid notch, the lateral wall of the mesiobuccal pulp chamber projected in the mesial direction. These results suggest that in preparation of cavities in mandibular first deciduous molars, close attention must be focused on pulp exposure in the pulp horn of the mesiobuccal pulp chamber. In addition, the lateral wall of the mesiobuccal pulp chamber should be noted in teeth with the deep, wide trigonid notch.
\end{abstract}

\author{
Key words \\ Mandibular deciduous molar, \\ Micro-CT, \\ Pulp chamber, \\ Three-dimensional observation, \\ Trigonid notch
}

\section{Introduction}

In deciduous teeth, it has been reported that the tooth substance (hard tissue) is thinner and the pulp horns are more pointed than in permanent teeth ${ }^{1}$. When coronal restoration of deciduous teeth is performed, appropriate cavity preparation with no pulp exposure is required in accordance with these characteristics. Therefore, in addition to the locations of the pulp horns, it is important to correctly understand the morphological properties of the deciduous crown and to be able to visualize the three-dimensional morphology of the inner pulp chamber from the

Received on September 30, 2009

Accepted on December 7, 2009 crown surface. It is also important to understand the thickness of the tooth substance at each pulp horn. However, because the contours of the crown of the mandibular first deciduous molars do not resemble those of the permanent teeth and other deciduous teeth, it is difficult to understand the morphology of the inner pulp chambers.

Studies on the topographic relationship between the contour of the crown and the pulp chamber have been performed, but observation has typically been two-dimensional and obtained via $\mathrm{X}$-rays ${ }^{2)}$ or sliced specimens ${ }^{3)}$, and three-dimensional structures have been difficult to elucidate. There have also been reports on three-dimensional observations and morphometry, but it has been difficult to obtain accurate three-dimensional reconstructed images and 
morphometric data because these methods involve a continuous series of sections examined by light microscopy and subsequently reconstructed. These methods are also destructive for the samples, and material losses occur during the preparation of sections ${ }^{4-6)}$.

Thickness of the tooth substance of deciduous teeth has been reported, but these papers were only related to the thickness of enamel or dentin ${ }^{7,8}$. Studies on the measurement of the distance from the enamel surface to the pulp chamber and metrical characteristics of coronal pulp chamber have been reported for the permanent teeth ${ }^{9,10)}$, but no such studies on deciduous teeth have been published.

Therefore, the crown contours and inner pulp chamber morphology were observed in three dimensions from various directions using micro-CT system, which has high resolution for crowns of the mandibular first deciduous molars and permits nondestructive three-dimensional observations from any direction. This study was focused on the morphology of the pulp chamber; especially the morphology of the pulp horns and their topographic relationships to the crown contour of the mandibular first deciduous molar. In addition, we attempted morphological analysis and made objective evaluations.

\section{Materials and Methods}

Study samples were 15 mandibular first deciduous molars. Teeth were macroscopically caries free with little attrition extracted from the dried skulls of Indian children in the deciduous dentition period on the occlusal line from the central deciduous incisor to the second deciduous molar. These teeth were scanned by using micro-CT system (HMX-225 Actis4, TESCO, Tokyo, Japan). The micro-CT system consists of an imaging device and a computer, which performs the calculations. The imaging device consists of an X-ray generator, a $360^{\circ}$ rotating specimen stage and an X-ray detector. During imaging, the tooth specimen was placed with the tooth axis vertical to the platform. Imaging conditions were as follows: tube voltage, $100 \mathrm{kV}$; tube current, $120 \mu \mathrm{A}$; focal spot size of $\mathrm{X}$-ray generator, $5 \mu \mathrm{m}$; magnification, $\times 10$; and slice thickness, $50 \mu \mathrm{m}$. The detector was equipped with an image intensifier measuring 4 inches and a 1 inch CCD camera with a scanning line of $1,024 \times 1,024$. The camera generated raw data comprising 250 two-dimensional images.

Three-dimensional reconstruction was performed using 250 two-dimensional images processed by TRI/3D-BON software (RATOC, Tokyo, Japan).

After different colors were applied to the pulp chamber, dentin and enamel in the three-dimensional reconstructed images to facilitate identification of each part, only the pulp chambers were sampled and observed from various directions. The enamel and dentin were rendered transparent and the topographic relationship between the contour of the crown and the pulp chamber was observed from various aspects.

The thickness of the tooth substance in each horn of the pulp chamber, optional sections in parallel with the tooth axis passing through the pulp horns were set on the three-dimensional reconstructed images. The distance between each pulp horn and enamel surface was calculated. The calculated value was analyzed among the related four groups by Friedman test which is a non-parametric test for a difference in central location (median) between two or more paired samples. A significant level was set at 0.01 .

\section{Results}

In this study 15 mandibular first deciduous molars were used for morphological observations. Most of the sample teeth, 11 of 15, had four cusps: the mesiobuccal cusp, mesiolingual cusp, distobuccal cusp and distolingual cusp. The other four had five cusps, with a distal cusp between the distobuccal cusp and distolingual cusp. However, this distal cusp was not located in the middle between the distobuccal cusp and distolingual cusp, but was adjacent to the distobuccal cusp. The heights of the mesiobuccal cusp and mesiolingual cusp were about the same, and these two cusps were higher than the distal cusps. Furthermore, near the cervical portion on the mesiobuccal surface of the crown, the projecting mesiobuccal ridge was clearly observed (Fig. 1).

One feature of the mandibular first deciduous molars was a trigonid notch observed between the mesiolingual cusp and mesiomarginal ridge. The shape of this trigonid notch was not uniform and a shallow, narrow type of trigonid notch (Fig. 2) and a deep, wide type (Fig. 3) were observed. In the deep and wide trigonid notch type, mesiodistal diameter of crown was longer than in the shallow, narrow notch type.

For detailed observation of the morphology of the 


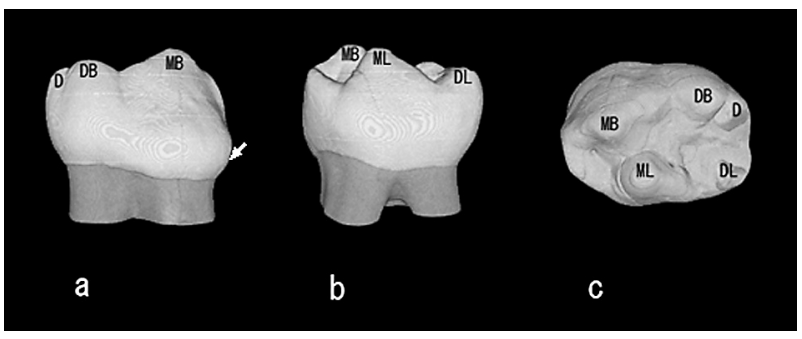

Fig. 1 3D rendered image of external crown morphology of mandibular first deciduous molar. Five cusps can be seen; the distal cusp was adjacent to the distobuccal cusp.

(a) Buccal aspect; (b) Lingual aspect; (c) Occlusal aspect MB: mesiobuccal cusp; DB: distobuccal cusp; ML: mesiolingual cusp; DL: distolingual cusp; D: distal cusp; arrow: mesiobuccal ridge

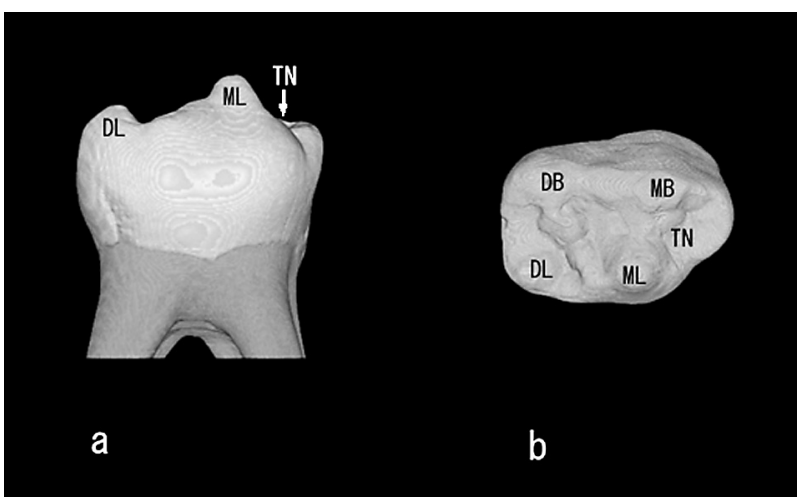

Fig. 2 3D rendered image of external crown morphology of mandibular first deciduous molar. The shape of trigonid notch is shallow and narrow.

(a) Lingual aspect; (b) Occlusal aspect

MB: mesiobuccal cusp; DB: distobuccal cusp; ML: mesiolingual cusp; DL: distolingual cusp; TN: trigonid notch

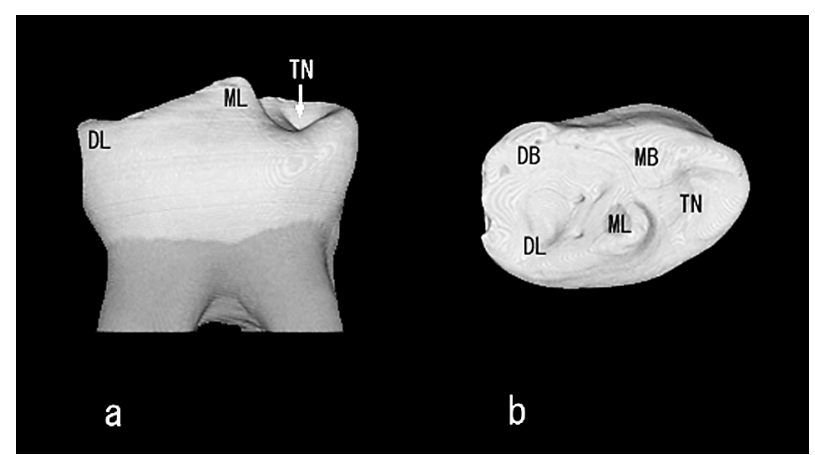

Fig. 3 3D rendered image of external crown morphology of mandibular first deciduous molar. The shape of trigonid notch is deep and wide.

(a) Lingual aspect; (b) Occlusal aspect

MB: mesiobuccal cusp; DB: distobuccal cusp; ML: mesiolingual cusp; DL: distolingual cusp; TN: trigonid notch

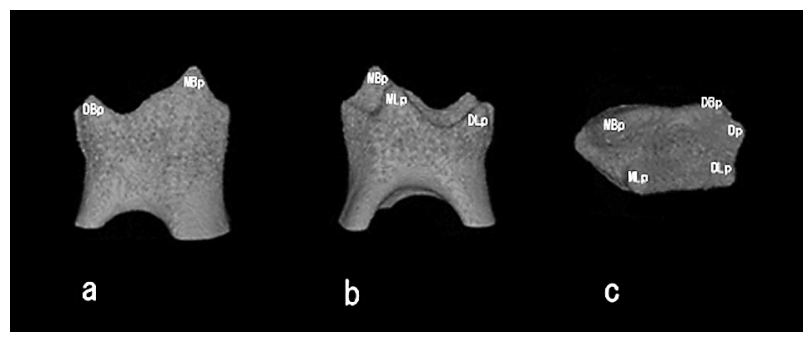

Fig. 4 3D rendered image of pulp chamber morphology of mandibular first deciduous molar (Corresponding to Fig. 1)

(a) Buccal aspect; (b) Lingual aspect; (c) Occlusal aspect MBp: mesiobuccal pulp horn; DBp: distobuccal pulp horn; MLp: mesiolingual pulp horn; DLp: distolingual pulp horn; Dp: Distal pulp horn

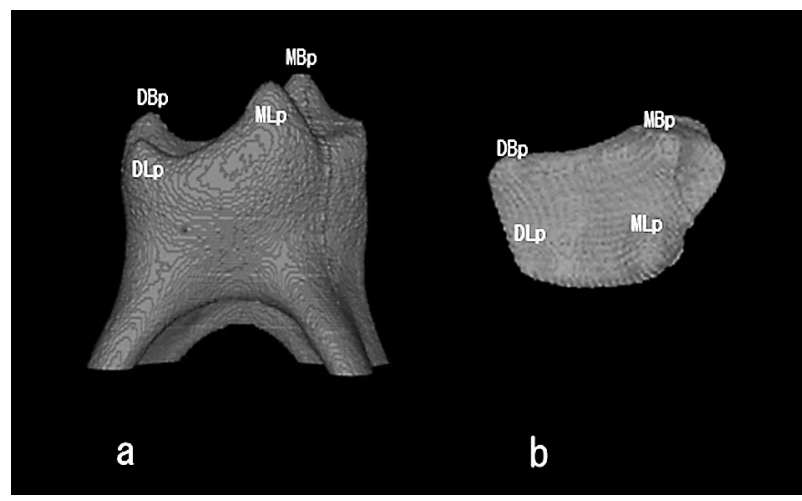

Fig. 5 3D rendered image of pulp chamber morphology of mandibular first deciduous molar (Corresponding to Fig. 2)

(a) Lingual aspect; (b) Occlusal aspect

MBp: mesiobuccal pulp horn; DBp: distobuccal pulp horn; MLp: mesiolingual pulp horn; DLp: distolingual pulp horn

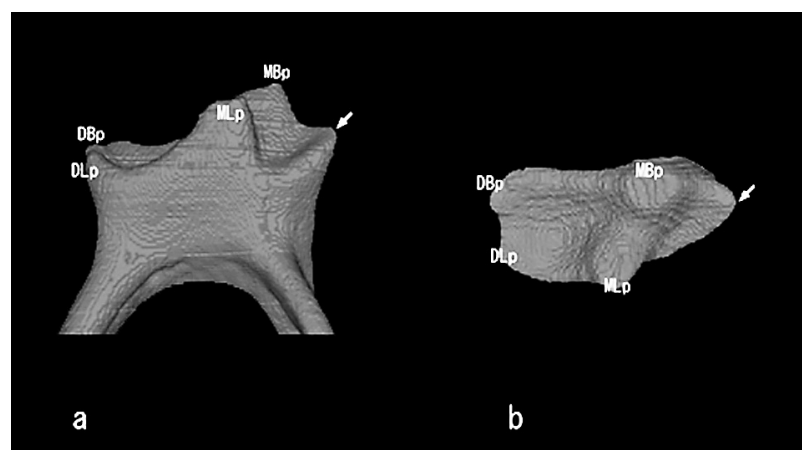

Fig. 6 3D rendered image of pulp chamber morphology of mandibular first deciduous molar (Corresponding to Fig. 3)

(a) Lingual aspect; (b) Occlusal aspect

MBp: mesiobuccal pulp horn; DBp: distobuccal pulp horn; MLp: mesiolingual pulp horn; DLp: distolingual pulp horn; arrow: eminent swelling of the mesial wall in the mesiobuccal pulp horn 


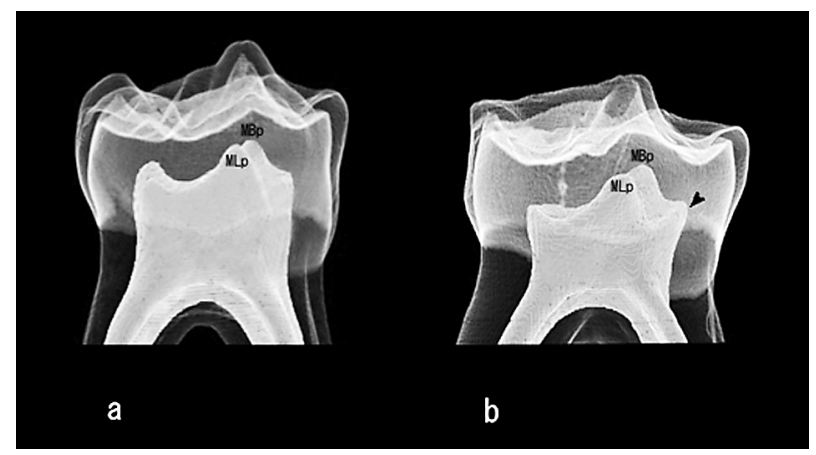

Fig. 7 3D topographic relationship between crown contour and pulp chamber from lingual aspect. In the deep, wide trigonid notch type (b), the lateral wall of the pulp horn at the mesiobuccal cusp showed marked projection (arrow head) on the mesial side and was closest to the enamel surface in this region.

(a) Shallow and narrow trigonid notch; (b) Deep and wide trigonid notch

inner pulp chambers, only the pulp chambers were obtained from the three-dimensional reconstructed images, and were observed from various directions. The morphology of the pulp chambers closely matched the crown contours. The number of pulp horns also matched the number of cusps, with four horns in teeth with four cusps and five horns in teeth with five cusps. The distal pulp horns corresponding to the distal cusps were also continuous with the distobuccal pulp horn and the size and height both decreased (Fig. 4, corresponding to Fig. 1).

Figure 5 shows the morphology of the pulp chamber in Fig. 2 (shallow and narrow trigonid notch type), while Fig. 6 shows that of the pulp chamber in Fig. 3 (deep and wide trigonid notch type). In both Fig. 5 and Fig. 6, the mesiobuccal pulp horn showed the greatest projection and the largest pulp horns. The lateral wall of the mesiobuccal pulp chamber also projected onto the medial side in Fig. 6.

In order to understand the topographic relationship of the crown contours and pulp chamber threedimensionally, the enamel and dentin, hard tissues covering the pulp chamber, were rendered transparent and observed from various directions. The results showed the greatest projection of the pulp horn in the mesiobuccal cusp and penetration in the direction of the cusp apex. Moreover, in the deep, wide trigonid notch type, the lateral wall at the mesiobuccal pulp chamber showed marked projec-

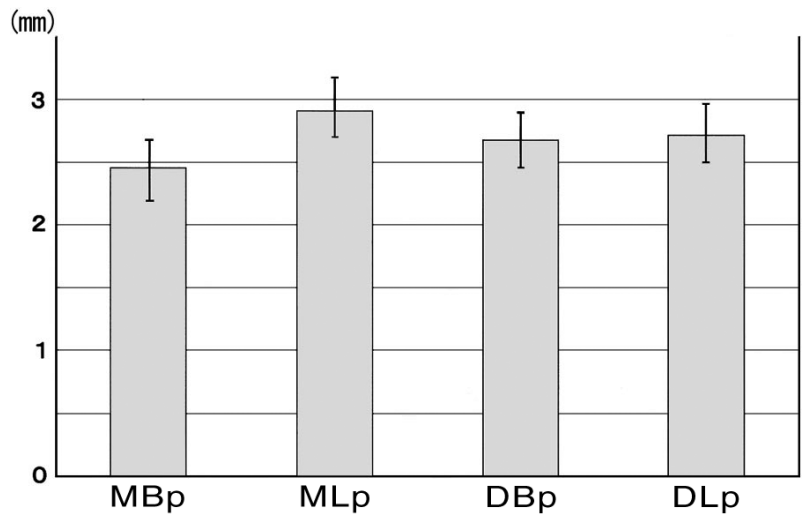

Fig. 8 A statistical significant difference was shown among the four related groups by Friedman test set at 0.01 . The mesiobuccal horn demonstrated a most decreased thickness.

MBp: mesiobuccal pulp horn; MLp: mesiolingual pulp horn; DBp: distobuccal pulp horn; DLp: distolingual pulp horn

tion on the mesial side and was closest to the enamel surface in this region (Fig. 7).

The thickness of the tooth substance (distance between pulp horn and enamel surface) at each pulp horn was as follows: mesiobuccal pulp horn (MBp), $2.45 \pm 0.30 \mathrm{~mm}$; mesiolingual pulp horn (MLp), $2.90 \pm 0.29 \mathrm{~mm}$; distobuccal pulp horn (DBp), 2.68 $\pm 0.21 \mathrm{~mm}$; distolingual pulp horn (DLp), $2.72 \pm$ $0.26 \mathrm{~mm}$. A significant difference on thickness of tooth substance was found among the four groups (Friedman test; $P<0.01$ ). The thickness in the mesiobuccal horn was less than that of the other regions (Fig. 8).

\section{Discussion}

To date, the internal morphology of teeth has generally been studied by means of radiographs ${ }^{2,9,10)}$ and serial sections ${ }^{6,11)}$. However, these methods had some problems such as unclear images or destructions of samples. Also, three-dimensional observation was required to obtain accurate data without destroying the specimens. Under these conditions, micro-CT system was introduced in this field. Then, this system has been widely used for the research works of the internal structure of root canals ${ }^{12-15)}$ that cannot be seen by direct vision. Recently, micro-CT system is also applied to understanding the morphology of the crown $^{16-18)}$. However, there have been few reports on three-dimensional observations of the pulp chamber 
in deciduous teeth or the topographic relationship between crown contours and the pulp chamber ${ }^{19,20)}$. Especially, the reports on mandibular first deciduous molars, which were used as samples in this study, have not been published.

Two-dimensional slice imaging data of horizontal sections obtained with a micro-CT system can clearly discriminate among enamel, dentin and pulp chambers with different levels of calcification. Therefore, the enamel, dentin and pulp chambers forming the dental crown are extracted independently and three-dimensional reconstruction is possible. This image also make possible to observe morphology from various directions and cutting through arbitrary planes.

In this study, the projection of the mesiobuccal horn and the lateral wall of the mesiobuccal pulp chamber projecting to the mesial side were observed from various directions.

Amano et al. ${ }^{19)}$ observed the morphology of the pulp chamber in maxillary second deciduous molars in the deciduous dentition and mixed dentition stages and reported greater changes in the morphology of the mesiolingual and distolingual pulp horns corresponding to the functional cusps. Therefore, the mandibular first deciduous molars in the present study were used in the deciduous dentition period in order to avoid the effects of attrition.

Philippas $^{21)}$ examined the effects of attrition and aging to the formation of the secondary dentin and the size of the pulp chamber in mandibular first molars. The results showed that aging had a greater effect than attrition on the formation of secondary dentin. Secondary dentin was not added uniformly to the inner surface of the dentin. The dentin at the floor of the pulp chamber and the mesial and distal walls of dentin increased in thickness with increasing age. However, attrition of the deciduous teeth progressed rapidly and addition of tertiary dentin was reported to be marked ${ }^{22}$.

Even though teeth without apparent attrition were used in this study, the height of the mesiobuccal pulp horns were significantly higher than that of the mesiolingual pulp horns. This result suggests the possibility that the mesiolingual cusp receives the functional force.

Deciduous teeth are said to have primitive characteristics. The trigonid notch expressed specifically in mandibular first deciduous molars is one of these primitive characteristics. Trigonid notches were observed in all 15 mandibular first deciduous molars used in this study, and these notches can be roughly classified into the deep, wide type and the shallow, narrow type. In teeth with the deep, wide trigonid notch, the inner pulp chamber is narrower on the buccolingual side and more extended mesiodistally than in teeth with the shallow notch. Moreover, the mesiobuccal pulp horn was wider mesially and projected mesially. Cavity preparation in pediatric dental treatment is generally made on mesio-occlusaldistal surface of the tooth crown. Therefore, it is necessary to pay attention to the mesiobuccal pulp horn and the morphology of the trigonid notch during cavity formation.

\section{Acknowledgments}

The authors would like to thank Professor Masatsugu Hashimoto (Department of Forensic Anthropology, Tokyo Dental College) for his critical editing and constructive comments and Professor Tsukasa Sano (Department of Oral and Maxillofacial Radiology, Tokyo Dental College) for conducting the statistical analysis.

\section{References}

1) Brand, R.D., Isselhard, D. and Stain, E.: Anatomy of Orofacial Structures. 7th ed. Mosby, St. Louis, 2003, pp.194-213.

2) Puddhikarant, P. and Rapp, R.: Radiographic anatomy of pulpal chambers of primary molars. Pediatr Dent 5: 25-29, 1983.

3) Arnim, S.S. and Doyle, M.P.: Dentin dimensions of primary teeth. J Dent Child 26: 191-214, 1959.

4) Mikrogeorgis, G., Lyroudia, K.L., Nikopoulos, N., Pitas, I., Molyvdas, I. and Lambrianidis, T.H.: 3D computer-aided reconstruction of six teeth with morphological abnormalities. Int Endod J 32: 88-93, 1999.

5) Lyroudia, K., Pantelidou, O., Mikrogeorgis, G., Chatzikallinikidis, C., Nikopoulos, N. and Pitas, I.: The use of 3D computerized reconstruction for the study of coronal microleage. Int Endod J 33: 243247, 2000.

6) Lyroudia, K., Mikrogeorgis, G., Bakaloudi, P., Kechagias, E., Nikolaidis, N. and Pitas, I.: Virtual endodontics: Three-dimensional tooth volume representations and their pulp cavity access. J Endod $\mathbf{2 8}$ : 599-602, 2002.

7) Grine, F.E.: Enamel thickness of deciduous and permanent molars in modern Homo sapiens. Am J Phys Anthropol 126: 14-31, 2005.

8) Ruschel, H.C. and Chevitarese, O.: A comparative study of dentin thickness of primary human molars. J Clin Pediatr Dent 27: 277-281, 2003. 
9) Scotti, R., Villa, L. and Carrosa, S.: Clinical applicability of the radiographic method for determining the thickness of calcified crown tissues. J Prosthet Dent 65: 65-67, 1991.

10) Chandler, N.P., Pitt Ford, T.R. and Monteith, B.D.: Coronal pulp size in molars: a study of bitewing radiographs. Int Endod J 36: 757-763, 2003.

11) Nozaka, K., Ono, R., Itoh, M., Satoh, T., Amari, E. and Nozaka, Y.: New cavity forms in first deciduous molars which maximize dentin thickness between cavity and pulp chamber. Bull Tokyo Dent Coll 31: 217-228, 1990.

12) Bergmans, L., Cleynenbreugel, J.V., Wevers, M. and Lambrechts, P.: A methodology for quantitative evaluation of root canal instrumentation using microcomputed tomography. Int Endod J 34: 390-398, 2001.

13) Peters, O.A., Schönenberger, K. and Laib, A.: Effects of four Ni-Ti preparation techniques on root canal geometry assessed by micro computed tomography. Int Endod J 34: 221-230, 2001.

14) Oi, T., Saka, H. and Ide, Y.: Three-dimensional observation of pulp cavities in the maxillary first premolar tooth using micro-CT. Int Endod J 37: 46-51, 2004.

15) Plotino, G., Grande, N.M., Pecci, R., Bedini, R., Pameijer, C.H. and Somma, F.: Three-dimensional imaging using microcomputed tomography for studying tooth macromorphology. JADA 137: 1555-1561,
2006.

16) Macchiarelli, R., Bondioli, L., Debénath, A., Mazurier, A., Tournepiche, J.-F., Birch, W. and Dean, C.: How Neanderthal molar teeth grew. Nature 444: 748-751, 2006.

17) Avishai, G., Muller, R., Gabet, Y., Bab, I., Zilberman, U. and Smith, P.: New approach to quantifying developmental variation in the dentition using serial microtomographic imaging. Microsc Res Tech 65: 263-269, 2004.

18) Hara, A., Sekiguchi, H. and Yakushiji, M.: Three dimensional observation of the occlusal grooves in the maxillary first and second deciduous molars with micro-CT. Ped Dent J 18: 102-115, 2008.

19) Amano, M., Agematsu, H., Abe, S., Usami, A., Matsunaga, S., Suto, K. and Ide, Y.: Three-dimensional analysis of pulp chambers in maxillary second deciduous molars. J Dent 34: 503-508, 2006.

20) Wong, F.S.L., Willmott, N.S. and Davis, G.R.: Dentinal carious lesion in three dimensions. Int $J$ Paediatr Dent 16: 419-423, 2006.

21) Philippas, G.G.: Influence of occlusal wear and age on formation of dentin and size of pulp chamber. $J$ Dent Res 40: 1186-1198, 1961.

22) Klinge, R.F.: A microradiographic and electron microscopic study of tertiary dentin in human deciduous teeth. Acta Odontol Scand 57: 87-92, 1999. 